According to Williams et al. three out of four patients who received cardiac transplants died of acute rejection 7,7 and 18 days after transplantation, although immunosuppressive agents were used. At post-mortem, no technical reasons could be found for the failure of the hearts, but some interesting histological changes were revealed. In two of the hearts the changes were consistent with the usual picture of acute rejectioninterstitial and perivascular round-cell infiltration, and some interstitial oedema and haemorrhage. The heart of the third and longest surviving recipient, however, did not conform so closely to this picture; it had an Arthus-like appearance with extensive interstitial haemorrhage and accumulation of polymorphonuclear leucocytes.

To assess the role of presensitization and circulating antibodies in the unusually virulent rejection processes experienced, immune adherence tests were performed with kidney (target) cells cultured from the donors. In the case of the second recipient, tests performed 10 days before transplantation provided evidence of pre-existing antibodies to antigens on the donor kidney cells. The fact that the same recipient serum did not react against either the lymphocytes or skin cells of the donor in the conventional pre-transplantation cross-match indicates the need for more sensitive and rapid tests to eliminate presensitization to donor antigens.

The antibody titre of the second recipient declined rapidly after grafting, but antibody was present in the eluate prepared from the rejected heart. This provides additional support for earlier observations that antibody is absorbed from the circulation by large organ grafts. Williams et al. say the quantity or quality of pre-existing antibody was probably insufficient to cause hyperacute rejection, but the acute rejection crisis is likely to have been a secondary, rather than a primary, response.

The most interesting finding-the presence of antibody fixed to an acutely rejected heart-was made in the case of the third recipient. Williams et al. showed that serum antibody directed against donor kidney antigens, which was not present before transplantation, appeared in low titre 12 days after transplantation and was present in high titre in the eluate prepared from the rejected heart. No antibody was detected in an eluate prepared identically from the patient's own kidney.

The exact role of humoral antibody in precipitating rejection of large organ grafts still remains unclear. Williams et al. suggest that the virulent form of rejection experienced may be a secondary response produced by an immunologically competent host, mediated by both cellular and humoral factors.

\section{BIOCHEMICAL DISEASE}

\section{Treutment by Vitumins}

\section{from our Medical Biochemistry Correspondent}

DURING the past two years, thirteen children have been described who were excreting large quantities of methylmalonic acid in their urine, although their diet was not deficient in vitamin $B_{12}$, a necessary factor in the metabolism of this acid. All the children suffered from vomiting, lethargy and ketoacidosis, and did not grow. Untreated, they were likely to die young, but some of them responded well to massive doses of $B_{12}$ and then grew and developed normally. Others, however, did not seem to be affected by the vitamin. Recent work has suggested that this inherited condition has at least two forms, and that the ability to respond to vitamin $B_{12}$ is present in only one form. Furthermore, the two forms can be distinguished in cultures of fibroblasts.

Vitamin $B_{12}$, in the form of deoxyadenosyl- $\mathrm{B}_{12}$, is necessary for the conversion of methylmalonyl coenzyme A to succinyl coenzyme A by methylmalonyl coenzyme A isomerase. Methylmalonyl coenzyme A is usually formed by carboxylation of propionyl coenzyme A, produced as a result of $\beta$-oxidation of oddnumbered fatty acids. Morrow et al. (Proc. US Nat. Acad. Sci., 63, 191; 1969) found that in liver homogenates prepared from patients with methylmalonicaciduria there was little conversion of propionate to succinate, and that three times as much methylmalonic acid accumulated as in control homogenates. The addition of deoxyadenosyl- $B_{12}$ to the homogenates increased the control values and brought one of the patients up to the range of the controls, but in three other patients there was no increascd activity.

When the conversion of methylmalonate to succinate was measured in the homogenates, the result was very similar. Three of the patients had very low enzyme activities in the liver, which were not increased by the addition of deoxyadenosyl- $\mathrm{B}_{12}$. But in the fourth patient's liver, enzyme activity increased to normal when the vitamin was added. This liver contained very little deoxyadenosyl- $\mathrm{B}_{12}$, but the others contained normal amounts. Morrow et al. therefore suggest that there are two forms of inherited methylmalonicaciduria, one in which the isomerase is inactive and which does not respond to vitamin $\mathrm{B}_{12}$, and the other which arises from a defect in $B_{12}$ metabolism or its binding to the isomerase and which responds to massive doses of $B_{12}$.

Rosenberg et al. (Biochem. Biophys. Res. Commun., $3 \%, 607$; 1969) have shown that this defect in metabolism can be studied in cultures of skin as well as liver. They found that fibroblasts from two patients with methylmalonicaciduria produced much less labelled carbon dioxide from labelled propionate and methylmalonate than fibroblasts from control patients. When deoxyadenosyl- $\mathrm{B}_{12}$ was measured in sonicates of fibroblasts a highly significant difference was revealed; the culture from one of the patients had $0.4-0.6 \mathrm{ng} / \mathrm{g}$ wet weight, while six control cultures contained on average $5 \cdot 9 \pm 2 \cdot 0 \mathrm{ng} / \mathrm{g}$ wet weight. When the $B_{12}$ in the medium was increased one-thousand-fold, normal amounts of coenzyme formed in the fibroblasts and the quantity of propionic acid oxidized was normal. These findings provide a convenient method for identifying methylmalonicaciduria, for it is obviously much easier to take skin than liver for culture.

\section{PROTEINS}

\section{Conservation and Divergence}

\section{from our Molecular Biology Correspondent}

Proteins vary widely in their capacity for absorbing mutations without detriment, and one supposes that 